\title{
Differential expression and structural polymorphism in rubber tree genes related to South American leaf blight resistance
}

\author{
Paula Cristina da Silva Angelo ${ }^{\mathrm{a}, *}$, Michel Eduardo Beleza Yamagishi ${ }^{\mathrm{b}}$, Jeferson Chagas da $\mathrm{Cruz}^{\mathrm{c}}$, \\ Gilvan Ferreira da Silva ${ }^{c}$, Luadir Gasparotto ${ }^{c}$
}

${ }^{a}$ Embrapa Café, IAPAR, CP 10.030, Londrina, PR, CEP 86057-970, Brazil

${ }^{\mathrm{b}}$ Embrapa Informática na Agropecuária, UNICAMP, CP 6041, Campinas, SP, CEP 18083-970, Brazil

${ }^{\mathrm{c}}$ Embrapa Amazônia Ocidental, CP 319, Manaus, AM, CEP 69010-970, Brazil

\section{A B S T R A C T}

South American leaf blight (SALB) is the worst impairment for rubber tree cultivation in the Amazon. Hevea brasiliensis clones produce high latex yields but most are SALB-susceptible (SS). So, resistance has been searched in related species. We selected defense related genes among those differentially expressed while contrasting SALB-resistant (SR) and susceptible clones from three Hevea species. SS clones over-expressed pathways responsive to salicylic (SA) and also jasmonic acid, thus entering a self-harmful cycle. SR clones displayed tightly coordinated SA-responsive and stress-control pathways. Polymorphism in the selected gene sequences supported SS $\times$ SR clone divergence.

\section{Introduction}

The rubber tree breeding program operated by the Brazilian Agricultural Research Corporation at Embrapa Western Amazon (Manaus, Amazonas State) employs Hevea brasiliensis, $H$. guianensis, $H$. pauciflora and $H$. nitida, among other species, for experimentation in grafting and hybridization, research which supports the recommendation of technologies such as inter-specific hybrids displaying high latex productivity in addition to South American leaf blight (SALB) resistance or SALB resistant crowns grafted on highly adapted tapping panels. SALB, caused by the fungi Pseudocercospora ulei (synonymy for Microcyclus ulei, is recognized as the principal biological barrier to large-scale commercial rubber tree cultivation in the Amazon biome [1,2].

$H$. brasiliensis clones can be considered the best latex producers, but they are caducous and susceptible to SALB, which can lead to precocious leaf decay and spoiled latex yields. In contrast, $H$. guianensis var. marginata and $H$. pauciflora are ever green plants, tolerant or resistant to SALB [1-4] despite most clones do not exhibit high latex yields. Besides $H$. guianensis and $H$. pauciflora, $H$. nitida genotypes do not lose healthy leaves seasonally [3] and, interestingly, some of the hybrids from these species are tolerant or resistant to the leaf blight [1].

$H$. brasiliensis is also the species in the genus more frequently assessed at the molecular level. A H. brasiliensis genome draft was recently accomplished [5]. A study on the differential expression of $H$. brasiliensis genes enrolled in plant-pathogen interaction during the first hours post-innoculation with $P$. ulei is recent as well [6]. Nevertheless, large scale databases for other Hevea species are scarce.

The activation of salicylic acid (SA) responsive reactions, hypersensitivity (HR), and epidermal cell damage associated to cyanogenesis has been reported as rubber tree defense reactions against $P$. ulei $[2,4,6]$. SA, and jasmonic acid (JA) and ethylene (ET) trigger plant defense reactions, following the recognition of MAMP (Microbe Associated Molecular Patterns, also designated as PAMP, for Pathogen Associated Molecular Patterns). MAMP recognition is the first line of plant defense, based on percepting the presence of chitin and other small molecules that interact with PRRs (Pathogen or Pattern Recognition Receptors). Reactive oxygen species (ROS) and calcium $\left(\mathrm{Ca}^{2+}\right)$ in the apoplast can also be part of MAMP and induce the production of SA and JA/ET, which is initiated in the chloroplasts. SA and JA subsequently interact with immediate downstream co-factors, which finally activate the transcription of cascades of defense related genes, and even the epigenetic remodeling of the chromatin, through histone modification. More commonly, SA is in charge of defense against biotrophic pathogens while JA/ET are involved in the reactions against herbivores and necrotrophic pathogens [see 7 for a recent review]. Nevertheless, despite SA and JA/ET pathways have been considered as mutual antagonists, the results of experimentation with mutants defective to one or more of these molecules (also designated as hormones) demonstrate that synergy can also be observed. The exposition of Arabidopsis to Penicillium chrysogenum dry mycelium induced SA and JA/ET responses simultaneously $[7,8]$.

\footnotetext{
* Corresponding author.

E-mail addresses: paula.angelo@embrapa.br (P.C.d.S. Angelo), michel.yamagishi@embrapa.br (M.E.B. Yamagishi), jeferson.cruz@embrapa.br (J.C.d. Cruz), gilvan.silva@embrapa.br (G.F.d. Silva), luadir.gasparotto@embrapa.br (L. Gasparotto).
} 
The objectives of this work were to compare $H$. brasiliensis to $H$. guianensis $+H$. pauciflora clones in order to identify differentially expressed genes (DEGs), subsequently selecting for those related to plantpathogen interaction. Leaves were collected in a region that is endemic for $P$. ulei and we considered that all the plants were equally challenged by the fungi. The metabolic processes effected by proteins encoded in 45 disease-related DEGs and their relationships to each other were assessed, to reveal interesting aspects of the interaction rubber trees - $P$. ulei, involving SA and JA-responsive pathways, under the additional stress represented by the extremely sunny equatorial climate. Finally, more than two thousands of polymorphic sites (SNPs, indels and repetitive element variations) were found in the sequences of those 45 disease related DEGs and paralog forms exclusive to the resistant or to the susceptible genotypes were identified.

\section{Material and methods}

Leaves in the B and C developmental stages, which are the stages when pathogen penetration-tissue colonization and sporulation can be observed, respectively [2], were collected at the main campus of Embrapa Western Amazon ( $\left.3^{\circ} 06^{\prime} \mathrm{S}-60^{\circ} 01^{\prime} \mathrm{W}\right)$, Manaus, Amazonas State, Brazil, within the region where Pseudocercospora ulei (Henn.)B.T.Hora \& Mizubuti (synonymy for Microcyclus ulei (Henn.)Arx) is endemic and there is high inoculum pressure [2]. The nine genotypes accessed through RNA-seq were Hevea brasiliensis (Willd. ex A.Juss.)Müll.Arg. clone RRIM 600 and clone RRIM 713, which are both susceptible to SALB [4,5] and the clone CNS AM 7905, an EMBRAPA breeding program selection that displays moderate resistance to SALB and is used as the tapping panel for grafting [1]; H. pauciflora Müll.Arg. clones CBA2, $\mathrm{PX}$ and PSB, which are resistant to SALB $[1,2,4] ; H$. guianensis var. marginata Ducke clones Hmg 1 and Hmg 2, which are resistant to SALB [1], and a $H$. guianensisAubl. $x H$. nitida Müll.Arg. (Hmg 17 x CPAA C 65) hybrid clone.

Total RNA was extracted using silica mini-columns, quantified in a micro-spectrophotometer, and evaluated in agarose gels. The cDNA libraries construction (paired ends) and NGS sequencing were accomplished in the LaCTAD facilities at the University of Campinas (São Paulo State, Brazil). Reads were mapped onto the $H$. brasiliensis reference genome [databases for FTP transference are cited in 5] using TopHat2 [9]. Transcripts were assembled by Cufflinks [10], and differential expression analyses were performed following the Cuffdiff protocol [11]. The expression level of the consensus transcripts in $H$. brasiliensis was compared to the expression level in $H$. pauciflora and $H$. guianensis. Additional analyses were performed on 327 transcripts identified through both comparisons as either up-regulated in both or down-regulated in both comparisons outputs.

In order to identify the metabolic pathways effected by the proteins they code for, the DEG sequences were uploaded to the BioSystems servers in the NCBI web site [12]. Orthologous Arabidopsis proteins [13] and associated Gene Ontology (GO) terms [14] were recovered in the BioSystems output files. This BioSystems output, the $H$. brasiliensis reference genome annotations, and the relevant literature were used as the basis to select 45 transcripts/encoded proteins involved in plantpathogen interaction out of that initial set of 327 .

Next, the 45 selected proteins were uploaded to the String servers [15], in order to investigate the interactions among them (physical interaction, co-occurrence, neighborhood, co-expression, and text mining in curated databases and literature bases) by clustering. Aiming to gather most of the 45 specimens in the major cluster, the confidence parameter for the interactions was set at 0.150 , and the maximal number of interactors was set at 75 in the first shell (direct interaction) and at 20 in the second shell. Clustering was performed using an MCL inflation parameter of 2 to enhance the production of smaller protein sub-clusters more specifically related to metabolic processes instead of to protein families/functions [16]. The String Analysis Pages included clusters of proteins representing different metabolic routes, the identification of the main GO biological processes assessed and the statistical significance $(\mathrm{P} \leq 0.05$ ) of the clusters produced, by comparing the number of expected edges to the number of observed edges at the end of the clustering routine. In addition to the initial analysis and clustering of most of the 45 disease-related DEGs, two additional analyses were performed, separately uploading to the String servers the 25 proteins that were up-regulated in $H$. brasiliensis and those 20 upregulated in $H$. guianensis $+H$. pauciflora.

Finally, for the 45 loci coding for the selected proteins, the consensus paralogs produced with reads from the nine rubber tree cDNA libraries were compared in order to identify species-specific structural polymorphisms. The polymorphisms were visualized using the IGV software (Broad Institute) and the IGV output was further examined by a human curator to find phenotype-specific (SALB resistant $\mathrm{x}$ susceptible) polymorphisms.

\section{Results}

The number of RNA-seq reads available from the nine Hevea transcriptomes ranged from 29,198 to $36,248 \times 10^{3}$. Bases displaying $\mathrm{Q} \geq 30$ were $95.03 \%$, on average. Assembling produced approximately 25,000 consensus transcripts. Based on read frequencies, the number of DEGs (p value for the $\log _{2}$ fold-change ranging from $5 \times 10^{-05}$ to $1 \times 10^{-4}$ ) was found to range between 2,000 and 5,000 when $H$. brasiliensis transcriptomes were contrasted with $H$. pauciflora and with H. guianensis separately.

Among those thousands of DEGs, 327 locus were present in the data sets resulting from both $(H$. brasiliensis $x H$. guianensis and $H$. brasiliensis $x$. pauciflora) comparisons, displaying one out of two opposite expression patterns: 1) up-regulation in $H$. brasiliensis and down-regulation in $H$. guianensis and $H$. pauciflora simultaneously, or 2) down-regulation in $H$. brasiliensis and up-regulation in $H$. guianensis and in $H$. pauciflora simultaneously. From those 327 , the group of 45 items in Table 1 was selected as defense related DEGs, according to the $H$. brasiliensis reference genome annotation and the BioSystems outputs. The heat map in the Suppl. Mat. S1 is a graphical representation of the two patterns of differential expression for the 45 selected DEGs. There were differences between the transcriptomes of $H$. guianensis and $H$. pauciflora (compare G x P in the Suppl. Mat. S1). However, those differences did not cause the disruption of pattern number 1 ( $B>G$ and B > P in the Suppl. Mat. S1, first 25 rubber tree DEGs) or number 2 (B $<$ G and B $<$ P in the Suppl. Mat. 1, last 20 DEGs). So, the values for the expression level (kfpm units) in Table 1 were calculated using RNAseq reads from the set of $H$. guianensis reads $+H$. pauciflora reads (the resistant clones/genotypes) to contrast with the reads from the set of $H$. brasiliensis clones/genotypes (the susceptible clones/genotypes). The direction of the changes in the expression level is indicated by the signals preceding the numerical values: a minus sign (-) indicates expression pattern number 1 and is observed for the first 25 items in Table 1, in agreement with the heat map in Suppl. Mat. S1. The opposite pattern, pattern number 2 , is indicated by a plus sign $(+)$, and was observed for the last 20 items in Table 1, in agreement with the heat map in Suppl. Mat. S1 as well. Despite the same DEGs were included in Table 1 and Suppl. Mat. S1, the ordination of the DEGs is not the same in those two information sources. This is because, for Table 1, the 45 entries were organized according to the fold-change values, while, for the heat map in Suppl. Mat. S1, entries were displayed in descending order according to the expression level in the $H$. brasiliensis transcriptome. The correspondence between the two information sources is made by the use of the ordination numbers taken from Table 1 (\# column) to identify the DEGs in the heat map (numbers in the left border of the heat map, Suppl. Mat. S1).

Homolog identity in the reference genome [5] and the BioSystems outputs diverged in a few cases, despite the fact that both identifications were made through the screening of Arabidopsis databases (see Suppl. Mat. S2 for the AOR coding sequence from the A. thaliana Ler 
Table 1

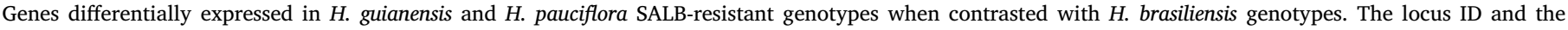

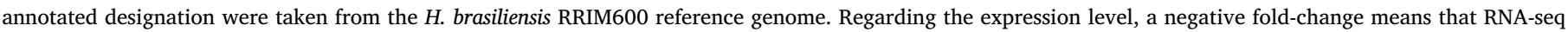

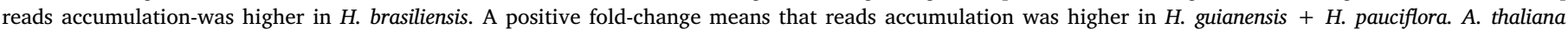

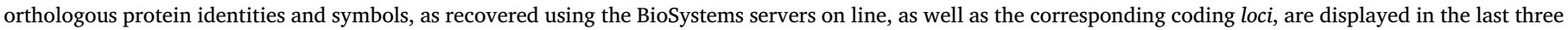
columns, respectively.

\begin{tabular}{|c|c|c|c|c|c|c|c|}
\hline \# & S-C $*$ & $\begin{array}{l}\text { H. brasiliensis } \\
\quad \text { locus }\end{array}$ & $H$. brasiliensis reference genome annotation & $\begin{array}{l}\text { Expression } \\
\text { level ** }\end{array}$ & $\begin{array}{l}\text { Best hit in } \\
\text { BioSystems }\end{array}$ & Protein symbol & $\begin{array}{l}\text { A. thaliana } \\
\text { locus ID }\end{array}$ \\
\hline 1 & $\mathrm{DCY}$ & 110647495 & EG45-like domain containing protein & -5.0898 & NP_001328443.1 & EXLB2 & AT4G30380 \\
\hline 2 & LTG & 110652792 & leucoanthocyanidin dioxygenase-like & -4.9456 & NP_194019.1 & LDOX & AT4G22880 \\
\hline 3 & PK & 110651561 & protein BOL4 chloroplastic/mitochondrial-like & -4.6818 & NP_197258.1 & BOLA4 & AT5G17560 \\
\hline 4 & PRP & 110641550 & valine $\mathrm{N}$-monooxygenase 1 & -4.5895 & NP_195705.1 & CYP79B2 (CYP79D2) & AT4G39950 \\
\hline 5 & & 110640229 & F-box/kelch-repeat protein At1g57790-like & -4.2583 & NP_001078341.1 & F-box/kelch-repeat & AT4G00893 \\
\hline 6 & $\mathbf{C Y}$ & 110634251 & protein TIFY 10A-like & -4.0081 & NP_564075.1 & JAZ1 & AT1G19180 \\
\hline 7 & $\mathbf{R}$ & 110650018 & bark storage protein A-like & -3.9145 & NP_-194166.2 & AT4G24350 & AT4G24350 \\
\hline 8 & DCY & 110673759 & peptidyl-prolyl cis-trans isomerase-like & -3.6322 & NP_179709.1 & AT2G21130 & AT2G21130 \\
\hline 9 & & 110652969 & amino acid permease 3 -like & -3.6168 & NP_-177862.1 & AAP3 & AT1G77380 \\
\hline 10 & $\mathbf{C Y}$ & 110638282 & protein TIFY 10B-like & -3.5167 & NP_565096.1 & TIFY 10B, JAZ2 & AT1G74950 \\
\hline 11 & $\mathbf{C Y}$ & 110651335 & pathogenesis-related protein 1-like & -3.3354 & NP_195098.1 & CAP (PR1) & AT4G33720 \\
\hline 12 & SBR & 110651577 & protein SIEVE ELEMENT OCCLUSION B-like & -3.0184 & NP_566145.1 & SEOa & AT3G01670 \\
\hline 13 & CY & 110632576 & protein TIFY 10A-like & -2.9425 & NP_001322261.1 & TIFY 10B, JAZ2 & AT1G74950 \\
\hline 14 & CY & 110639420 & protein TIFY 9-like & -2.9296 & NP_568287.1 & TIFY 9, JAZ10 & AT5G13220 \\
\hline 15 & DCY & 110651686 & probable LRR receptor like serine/threonine & -2.6800 & NP_o01154317 & RLP1 & AT1G07390 \\
\hline 16 & LTG & 110648892 & chalcone synthase 2 & -2.5303 & NP__196897.1 & ATCHS,TT4 & AT5G13930 \\
\hline 17 & PK & 110645775 & chitin-inducible gibberellin-responsive protein 1-like & -2.2600 & NP_-199626.1 & PAT1 & AT5G48150 \\
\hline 18 & & 110632326 & mitochondrial phosphate carrier protein 1 mitochondrial & -2.0200 & NP_181325.2 & ATG237890 & AT2G37890 \\
\hline 19 & LMG & 110652409 & ubiquitin-60S ribosomal protein L40 & -2.0097 & NP_ $\overline{00} 1190681.1$ & UBQ11 & AT4G05050 \\
\hline 20 & LMG & 110665745 & ubiquitin-conjugating enzyme E2 22-like & -1.8383 & NP_849462.1 & UBQ9 & AT4G27960 \\
\hline 21 & $\mathbf{R}$ & 110669030 & UDP-glycosyltransferase 74E1-like & -1.7284 & NP_ $\overline{00} 1184915.1$ & AT1G05675 & AT1G05675 \\
\hline 22 & $\mathbf{R}$ & 110640241 & uncharacterized LOC110640241 & -1.7132 & NP_190291.1 & AT3G47070 & AT3G47070 \\
\hline 23 & $\mathbf{C Y}$ & 110640442 & defensin-like protein & -1.6208 & NP_178319.1 & LCR69 (PDF 1.2) & AT2G02100 \\
\hline 24 & $\mathbf{Y}$ & 110639362 & beta-glucosidase 24-like & -1.5652 & NP_-181976.1 & BGLU17 & AT2G44480 \\
\hline 25 & $\mathbf{R}$ & 110672955 & ABC transporter B family member 27-like & -1.5365 & NP_001332027.1 & $\mathrm{ABCB} 27$ & AT5G39040 \\
\hline 26 & LTG & 110648442 & BAHD acyltransferase DCR-like & 1.3144 & NP_195741.1 & AT5G01210 & AT5G01210 \\
\hline 27 & B & 110639465 & CBL-interacting serine/threonine-protein kinase 14-like & 1.4891 & NP_195802.1 & CIPK14 (SR1) & AT5G01820 \\
\hline 28 & LMG & 110631454 & RING-H2 finger protein ATL2-like & 1.8896 & NP_-188294.1 & ATL2,TL2 (RIN2) & AT3G16720 \\
\hline 29 & $\mathbf{R}$ & 110638453 & cyclic nucleotide-gated ion channel 4-like & 1.8931 & NP_200236.1 & CNGC4 & AT5G54250 \\
\hline 30 & $\mathbf{R}$ & 110657526 & 2-methylene-furan-3-one reductase-like & 2.0801 & OAP 18379.1 & AOR (Ler) & AXX17-AT1G24910 \\
\hline 31 & B & 110638064 & homeobox-leucine zipper protein ATHB-12-like & 2.3210 & NP_191748.1 & HB-12 & AT3G61890 \\
\hline 32 & $\mathbf{R}$ & 110659237 & No Hits in 2013 & 2.5428 & NP_192162.2 & AT4G02530 & AT4G02530 \\
\hline 33 & BR & 110633217 & probable xyloglucan endotransglucosylase/hydrolase ptn 8 & 2.5680 & NP_563892.1 & XTH8 & AT1G11545 \\
\hline 34 & LMG & 110632206 & protein NRT1/PTR FAMILY 4.6-like & 2.6356 & NP_564978.1 & NRT1:2 & AT1G69850 \\
\hline 35 & B & 110636735 & probable pectate lyase 13 & 2.6548 & NP_-191052.2 & PMR6 & AT3G54920 \\
\hline 36 & LTG & 110645761 & protein SRG1-like & 2.8579 & NP_-173145.1 & SRG1 & AT1G17020 \\
\hline 37 & $\mathbf{R}$ & 110652954 & mechanosensitive ion channel protein 10 -like & 2.9519 & NP_-196769.1 & MSL10 & AT5G12080 \\
\hline 38 & B & 110649647 & plasma membrane ATPase 4 & 3.0294 & NP_-194748.1 & HA2 & AT4G30190 \\
\hline 39 & BR & 110647415 & polygalacturonase inhibitor-like & 3.0450 & NP_196304.1 & PGIP1 & AT5G06860 \\
\hline 40 & B & 110637485 & CCG-binding protein 1 -like & 3.1583 & $\mathrm{NP}^{-} 565383.1$ & MEE14 & AT2G15890 \\
\hline 41 & LTG & 110650461 & peroxidase 64 -like & 3.8330 & NP_199033.1 & PER64 & AT5G42180 \\
\hline 42 & PRP & 110672946 & isoflavone 3'-hydroxylase-like & 3.8568 & NP_568533.2 & CYP81D1 & AT5G36220 \\
\hline 43 & $\mathbf{R}$ & 110652570 & L-type lectin-domain containing receptor kinase IV.1-like & 3.9284 & NP_-181307.1 & RLK1, LRK1 & AT2G37710 \\
\hline 44 & $\mathbf{Y}$ & 110652529 & (S)-hydroxynitrile lyase-like & 4.7648 & NP_-196592.1 & MES5 & AT5G10300 \\
\hline 45 & G & 110645525 & histone H2B-like & 5.9696 & NP_200799.1 & HTB4 & AT5G59910 \\
\hline
\end{tabular}

ecotype and the 2-methylene-furan-3-one reductase-like sequence from the Col ecotype referring to item 30 homologs). This certainly occurred because during the six or seven years from the publication of the $H$. brasiliensis reference genome [5] and the database screenings made for the present work, data have been constantly uploaded, creating the opportunity to hit additional homologs to rubber tree sequences. Besides that, the screening of Arabidopsis protein databases (the BioSystems screening for this work) instead of non-redundant protein databases (the screening for the reference rubber tree annotation [5]) can result in discrepancies. Non reduntant databases include Arabidopsis proteins and a huge amount of deduced peptides from other plant species. Nevertheless, the BioSystems and the String (see below) routines are available for a limited number of species and Arabidopsis was the best choice, since it is a dicotyledon and maybe the species better explored to model plant-pathogen interactions, and this grants the access to constantly updated, informative databases. Discrepancies were commented in the sub-sections of the Results, when it was relevant to comprehend the information in the text.

So, following the BioSystems identification of the best Arabidopsis orthologs for the proteins encoded in 327 DEGs, the association of the proteins with Gene Ontology (GO) terms and the selection of those 45 defense related DEGs/encoded proteins which are included in Table 1, the clustering was performed using the String routines (output is in Fig. 1 and see also Suppl. Mat. S3 for the Analysis Page). As an aid to the BioSystems analysis, String clustering was important to show how those 45 proteins interacted to accomplish different metabolic processes, justifying and supporting their association to ascertained GO terms. The 45 entries in Table 1, which are identified by the same formal symbols 


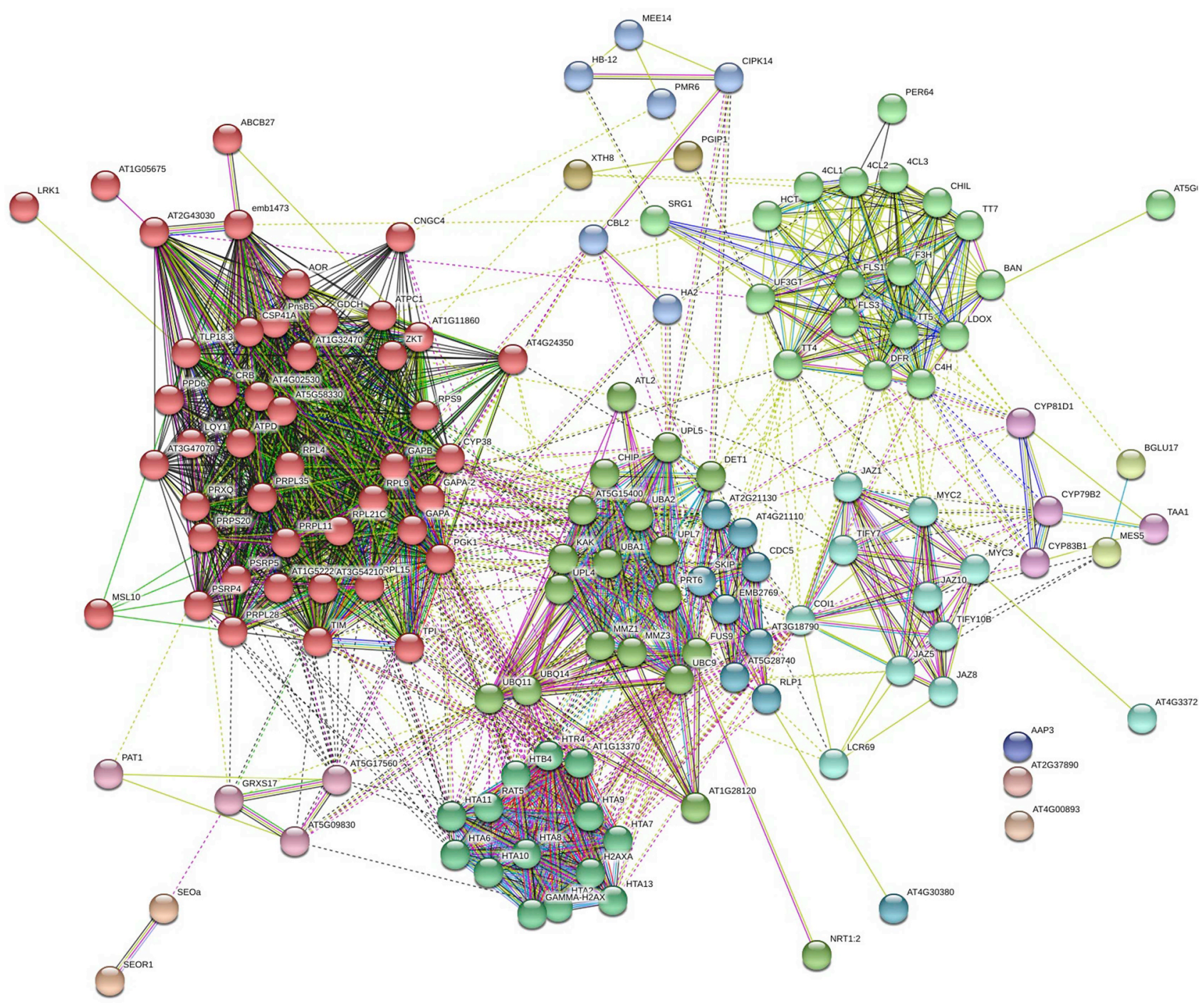

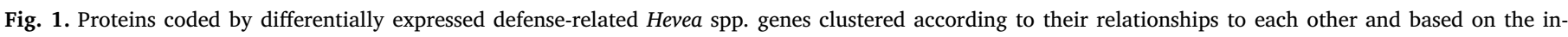

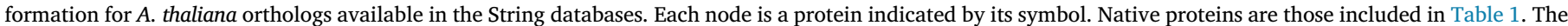

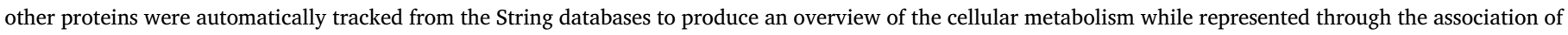

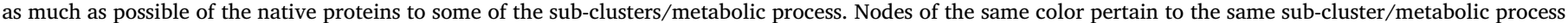
(see details in Supp. Mat. S3).

and/or loci designations (Table 1, Protein symbol) in Fig. 1, are henceforth referenced as "native" to the present study. In addition to these 45 "native nodes", Fig. 1 included proteins automatically tracked from the A. thaliana String databases due to the existence of data attesting for their direct or indirect interactions with the native ones. The automatically tracked non-native proteins/nodes are, as well, identified by their formal symbols, which can be used to recover additional information about them (see also Suppl. Mat. S3A with the legends for the String clustering output).

Indeed, the protein sub-clusters/groups of nodes in Fig. 1 were automatically displayed with different colors to distinguish the metabolic processes they are enrolled in (see Suppl. Mat. S3 and S3A, Analysis and Legend pages, for the identification and statistical significance of the sub-clusters representing the principal physiological processes that were assessed during clustering). With the exception of items 5, 9, and 18, all of the proteins in Table 1 joined one of the 12 sub-clusters of nodes in Fig. 1 (see Table 1, S-C). To construct a wide picture of the leaf metabolic status based on the relationships of as many as possible of the proteins encoded in selected rubber tree DEGs (Table 1), the major cluster (Fig. 1) was supported by more than a thousand edges. It is not possible to comment about thousands of relationships in a same study. In this reason, the proteins native to the present work (Table 1) were taken as focal points to comment, in the next section, the sub-clusters they joined to. Frequently, but not always, proteins encoded in native DEGs that followed the expression pattern number 1 (Table 1 and Suppl. Mat. 1 items 1 to 25, see explanation above) and those encoded in DEGs that followed expression pattern number 2 (Table 1 and Suppl. Mat. 1 items 26 to 45) were automatically included in a same sub-cluster (Fig. 1 and Table 1 S-C column), because they take part in a same metabolic process. The occurrence of these admixtured sub-clusters, in addition to the existence of sub-clusters gathering exclusively proteins coded by DEGs that followed expression pattern number 1 or pattern number 2 , contributed to comprehend how the leaf cells in plants of the different rubber tree genotypes were pushed to opposite reactions to $P$. ulei presence, and to exhibit contrasting phenotypes of susceptibility or resistance.

The major cluster in Fig. 1 (specimens 1 to 45) was subsequently divided into two representations of metabolic processes accomplished only by proteins up-regulated in the $H$. brasiliensis clones (specimens 1 to 25 in Table 1, Suppl. Mat. S4) or just by proteins up-regulated in the $H$. guianensis $+H$. pauciflora resistant clones together (specimens 26 to 45 in Table 1, Suppl. Mat. S5), to construct theoretical scenarios, ignoring down- 
regulated proteins (specimens 26 to 45 for Suppl. Mat. S4 and 1 to 25 for Suppl. Mat S5) as if they were not expressed at all. These theoretical scenarios were useful to estimate the consequences, e.g., of silencing downregulated proteins in each of the two sets of rubber tree clones/genotypes.

The second section of the Results is about the structural polymorphisms found in the transcripts produced with reads from the different RNA-seq libraries.

\subsection{Metabolic pathways running in the rubber tree leaf cells collected for RNA-seq}

\subsubsection{Membrane proteins and processes occurring in the organelles}

The proteins in the red sub-cluster in Fig. 1, according to the associated GO terms, are all related to processes that take place in the organelles (chloroplasts in particular) and involve trans-membrane trafficking.

The native members in the red sub-cluster (Table $1, \mathrm{~S}-\mathrm{C}=\mathrm{R}$ ) upregulated in $H$. brasiliensis and down-regulated in $H$. pauciflora $+H$. guianensis, and so following the expression pattern number 1 (see above), were items 7 (ortholog to AT4G24350), 21 (AT1G05675), 22 (AT3G47070), and 25 (ABCB27). These four proteins lack characterization even in $A$. thaliana and, for this reason, the BioSystems output for three out the four only included the homologous loci identities. In addition, proteins 21 (AT1G05675) and 25 (ABCB27) did not cluster with those up-regulated in $H$. brasiliensis (Suppl. Mat. S4). Item 18 by its turn was up-regulated in $H$. brasiliensis as well but did not cluster at all (Fig. 1, down in the right corner). Absence of edges tracking these native proteins to a sub-cluster may just mean that an even higher order of interactions would be necessary to attract them. On the other hand, maybe these proteins have roles in metabolic routes not sufficiently explored. Intriguingly, when those three "outliers" up-regulated in the susceptible genotypes (items 18, 21 and 25) were uploaded to the String servers as an independent set of baits for clustering, a tiny protein cluster associated with the metabolic process designated as "ribosomal sub-unit export from nucleus" (GO:0000054) was produced, including a proteasome activating protein (PA200, Suppl. Mat. S6). Ribosomal subunits are assembled in the nucleoplasm through the joining of rRNA and proteins imported from the cytoplasm. Assembled sub-units are then exported back into the cytoplasm through the recruitment of transporters [17], a process which occurs during apoptosis [18]. Despite ABCB27 joined the cluster of proteins in Suppl. Mat. S6, involved in "ribosomal sub-unit export from nucleus", reports on the specific participation of ATP-binding transporters in such a process could not be recovered. According to the homologous locus AT5G39040 description in Arabidopsis, the rubber tree ABCB27 would more likely be involved in the transportation of $\mathrm{Fe}-\mathrm{S}$ clouds in mitochondria.

Taking all this together, it can be considered that susceptible rubber tree plants were going through organelle recycling and non-programmed cell death (apoptosis).

By their turn, also in the red sub-cluster (Table 1, S-C = R), items found to be up-regulated in $H$. pauciflora $+H$. guianensis and downregulated in $H$. brasiliensis, in opposition to the proteins described in the previous paragraph, were the homologs to CNGC4 (encoded in transcript 29, Table 1), AOR (encoded in transcript 30, corresponding to the locus AXX17-AT1G24910 ecotype Landsberg Erecta, bioproject PRJNA311266 in the NCBI), AT4G02530 (item 32), MSL10 (item 37), and LRK1 (item 43).

CNGC4 codes for a plasma membrane calmodulin-binding protein, which is also known as DND2 for "defense, no death" and HML1 as well. It is associated with the "plant-type hypersensitive response" (HR) [19]. CNGC4 can homodimerize or heterodimerize with CNGC2, its closest paralog, to form cation gates that actively transport $\mathrm{Ca}^{2+}$ across the plasma membrane. Though their development of the HR response is impaired, A. thaliana cngc4 loss-of-function mutants exhibit autoimmune phenotypes, with constitutive expression of pathogenesis-related genes, elevated levels of SA, and, in some circumstances, the production of spontaneous HR-like lesions in the absence of pathogen infection or when infected by avirulent pathogens [20].

AOR is involved in the initial steps of reactive carbonyl detoxification in chloroplasts. Short-chain carbonyls are produced by stress-triggered oxidation of membrane-bound long-chain fatty acids, such as linoleic and linolenic acids, and could harm the photosynthetic apparatus [21].

MSL10 is associated with $20 \mathrm{GO}$ terms, such as programmed cell death in response to ROS (GO:0097468), detection of mechanical stimulus (GO:0050982), gated channel activity (GO:0022836), and substrate-specific trans-membrane transporter activity (GO:0022891), in addition to "phyllome development" (GO:0090693, see additional information below). MSL10 could serve as an early signal of wounding [22]. Resembling CNGC4 described previously, MSL10 participates in ion-gated transport and programmed cell death induction as separate activities. The protein amino-terminal (N-terminal) domain can induce cell death in response to ROS accumulation. The protein carboxyterminal (C-terminal) domain participates in the assembly of a mechanosensitive channel, which can respond to osmotic stresses that cause the plasma membrane to stretch [23]. The C-terminal could stabilize the $\mathrm{N}$-terminal portion, avoiding spontaneous cell death in nonwounded plants [22].

LRK1 is a serine/threonine kinase that contains extracellular lectin motifs resembling legume soluble (L-type) lectins, which may serve in the recognition of small hydrophobic ligands, such as MAMP-related molecules. Among the L-type kinases, $H$. brasiliensis reference locus 110652570 (item 43) was identified as a LecRK-IV.1 ortholog, which displays extremely high activity $52 \mathrm{~h}$ after exogenous SA application [24].

So, the red sub-cluster native proteins which were up-regulated in the resistant genotypes, indeed following the expression pattern number 2 (see above), have roles in the fast and effective detection of the pathogen employing trans-membrane MAMP-recognizers, and in disciplined (non-constitutive) production and response to SA, prompting the cells for defense based in precisely programmed cell death coordinated with the restrain of the oxidative stress at defenseuseful levels. The lack of these controlling mechanisms probably led susceptible plant cells to apoptosis and organelle recycling, as mentioned above.

The protein encoded in transcript 32, the last native member of the red sub-cluster, codes for a poorly characterized protein expressed in the chloroplast thylakoid lumen.

\subsubsection{Ubiquitination versus hypersensitive response}

The lime green sub-cluster, in the center of Fig. 1, is populated with proteins involved in ubiquitination and ligation of proteins to ubiquitin. The native proteins (Table 1, S-C = LMG) included in this sub-cluster which are expressed in higher levels in $H$. brasiliensis are UBQs 11 and 9 (items 19 and 20 in Table 1, respectively). The ubiquitination process involves covalent attachment of the highly conserved small protein ubiquitin to substrate proteins through a step-wise enzymatic cascade typically catalyzed by three different classes of enzymes: ubiquitin-activating enzyme (E1 or UBA), ubiquitin-conjugating enzyme (E2 or UBC), and ubiquitin ligase (E3), which transfers ubiquitin to the substrate. Conventionally, ubiquitination serves as the principal signal for protein degradation by the $26 \mathrm{~S}$ proteasome [reviewed in 25]. Ubiquitination interacts with the autophagy of larger non-proteinaceous structures of cells, such as ribosomes [26], and this connects the red and the lime-green sub-clusters of proteins and the metabolic routes they represent in $H$. brasiliensis.

Meanwhile, proteins in the lime-green sub-cluster that were upregulated in $H$. pauciflora $+H$. guianensis are involved in $\mathrm{HR}$ (GO:0010363) and $\mathrm{Ca}^{2+}$ transportation, just as those in the red subcluster (see above). According to the BioSystems output, the best hit for item 28 (Table 1) was ATL2, which is another MAMP-recognizer that responds to chitin [27]. Nevertheless, the best hit for the conserved 
domains in the ortholog protein was to RPM1-interacting protein 2 (RIN2), which is involved in the positive regulation of the HR and also in the ubiquitin-dependent protein catabolic process (GO: 0006511), fitting better to the annotation as a defensin (Table $1, H$. brasiliensis reference genome annotation). RIN2 from A. thaliana and the rubber tree protein encoded by item 28 display the same C3HC4-RING type zinc finger and CUE ubiquitin component domains. RIN2 exerts localized E3 ubiquitin-ligase/transferase activities, while its partner, RPM1, is a CC-NB-LRR protein that recognizes pathogens by their specificities and induces rapid ion fluxes, sustained oxidative bursts, and a basal defense transcriptome, often accompanied by localized programmed cell death at the infection site [28]. The C-terminal of RIN2 (and RIN3) is involved in the interaction with RPM1, as well as in RIN2-RIN3 homo- or heterodimerization [29], which is necessary to accomplish the transfer of ubiquitin to substrate proteins. Thus, a decrease in the availability of ATL2/RIN2-like proteins could protect RPM1 from degradation, inducing the downstream defense reactions, including cyanogenesis, to be primed. Kawazaki et al. [29] reported this hypothesis but could not fully demonstrate it. Considering the reduction in gene expression by almost two folds in $H$. brasiliensis relative to $H$. guianensis $+H$. pauciflora (Table 1 ), a decrease in the availability of the ATL2/RIN2 protein and the priming of defense reactions can be anticipated in the first genotypes.

NTR1:2 (coded in transcript number 34, Table 1) is an integral plasma membrane transporter that responds to $\mathrm{Ca}^{2+}$ and moves nitrate and abscisic acid (ABA) molecules non-competitively [30], a process which may modulate growth rhythms according to the needs to activate or not defense related stress-control.

Resistant rubber trees would, so, have more than a set of up-regulated genes involved in pathogen recognition and ion movement across membranes, because proteins in the lime-green sub-cluster (Table 1, S$\mathrm{C}=\mathrm{LMG}$ ) would join those in the red sub-cluster (Table $1, \mathrm{~S}-\mathrm{C}=\mathrm{R}$ ) to trigger tightly controlled defense reactions. Meanwhile, growth, or at least photosynthesis and the primary metabolism, would not be put aside, if the influx of ABA was in tune with the efflux of $\mathrm{Ca}^{2+}$ to the apoplast, where it can contribute to characterize MAMP (and additional physiological processes that are described below). Instead, in susceptible genotypes, the slower or mild MAMP-recognition due to the downregulation of RLK1 and ATL2/RIN2 and MSL10 would aid the decreased expression of CNGC4, in the arise of what could be considered a delayed and disorganized excessive defense reaction.

\subsubsection{Cell-cell junction, cell wall modification and cytoplasm detoxification} are up-regulated in $H$. guianensis $+H$. pauciflora

With the exception of CBL2 that was tracked from the String databases, the small blue sub-cluster (Table $1, \mathrm{~S}-\mathrm{C}=\mathrm{B}$ ) in the upper corner of Fig. 1 consists entirely of members native to the present study, which were up-regulated in the resistant $H$. guianensis $+H$. pauciflora genotypes. The two members of the brown sub-cluster (Table $1, \mathrm{~S}-\mathrm{C}=\mathrm{BR}$ ) that intersected the blue sub-cluster in Fig. 1 followed exactly the same trend. So, the blue and the brown sub-clusters are populated exclusively with proteins encoded in DEGs that followed expression pattern number 2.

Proteins HB-12, PMR6, and MEE, encoded in transcripts 31, 35, and 40 in Table 1, are involved in biological processes such as "response to biotic stimuli" (GO:0009607), "response to fungi" (GO:0009620), and "response to chitin" (GO:0010200). HB-12 is a leucine zipper protein that responds to oxidative stress, water stress, and abscisic acid. The coordinated expression of HB-7 and HB-12 could modulate growth in the context of water stress [31]. In A. thaliana, PMR6 is involved in JA/ SA-independent resistance mechanisms and exhibits pectate-lyase enzymatic activity, which is important for cell wall properties modifications [32] and drought tolerance [33]. MEE proteins have DNA binding domains and are most likely involved in DNA repair in the context of rubber tree leaves considering the description of its characteristics in Arabidopsis.
Still related to the cell wall, in the brown sub-cluster, item 33 encodes a xyloglucan hydrolase, and item 39 encodes an ortholog to PGIP1, a polygalacturonase inhibitor protein, which inhibits pectindegrading enzymes produced by fungal pathogens. PGIP has been demonstrated to be up-regulated upon infection of $A$. thaliana by Stemphylum solani [34].

Back to the native proteins in the blue sub-cluster, item 27 from Table 1 code for the ortholog to A. thaliana CYPK14 in rubber trees. CYPK14 takes part in the $\mathrm{Ca}^{2+}$-dependent signal transduction mechanism, forming pairs with calcineurin B-like (CBL) proteins. CBLs could be sensors of $\mathrm{Ca}^{2+}$ concentration and CIPKs, the signal transductors. CBL/CIPK complexes, which are present in a variety of cell membranes, regulate calcium storage and release into the cytoplasm, and import and export to the apoplast, the tonoplast/vacuole, the endoplasmic reticulum, the mitochondria, and the chloroplasts. Specifically, CBL2/CIPK14 complexes were found to be located in the A. thaliana tonoplast membrane [35]. It will be interesting to search for the rubber trees to CBL partner of the CIPK14, but we can already speculate that the $\mathrm{Ca}^{2+}$-responsive pathways would include CNGC4, MSL10, and NTR1:2, which joined the red and the lime-green subclusters.

Item 38 by its turn code for a plasma membrane-ATPase associated with the GO terms "cell-cell junction" and "symplast", and designated by the symbol HA2. HA2 is an integral membrane protein that may also be located in the internal membrane system, including the Golgi apparatus and the vacuolar membrane, or in plasmodesmata membranes, according to the corresponding Arabidopsis locus (Table 1) description. It is important for the symport of molecules accompanied by $\mathrm{H}^{+}$cations. HA1 and HA2 are paralogs and apparently play redundant functions in most plant organs, though HA2 expression has been primarily studied in the roots. ha2 mutants display reduced growth when subjected to high external $\mathrm{pH}$ or potassium concentrations and grow better than wild-type plants when exposed to toxic cations and other intoxicating molecules, such as amino-glucoside antibiotics. Nevertheless, when the $\mathrm{H}^{+}$transportation is reduced by an increased external concentration of potassium, imports of toxic molecules are reduced in wild-type plants [36].

Is it possible that the $\mathrm{Ca}^{2+}$ ions in the resistant genotypes play a role similar to that of potassium [37] and contribute to the prevention of cellular intoxication during cyanogenesis? If the consequences of the three-fold decrease in HA2 expression observed for $H$. brasiliensis (Table 1) can be compared to the ha2 loss of function mutations reported for A. thaliana [38], than it can be considered that these two genotypes would grow better under intoxicating conditions than their counterparts in the same genus. Meanwhile, $H$. guianensis $+H$. pauciflora would rather be prepared to control and limit inter-cellular pectin degradation and water loss resulting from fungi exploration of the stomatal cells by moving $\mathrm{Ca}^{2+}$ among the different cellular compartments. The increase in extra-cellular $\mathrm{Ca}^{2+}$ driven by NTR1:2 (Table 1 , item 34, lime green sub-cluster) would facilitate the import of ABA from the apoplast to spread stomatal closure, balancing growth and defenserelated metabolic pathways. And, maybe, $\mathrm{Ca}^{2+}$ in the apoplast would assure that free $\mathrm{HCN}^{-}$an other intoxicating molecules produced outside the cell membrane (see below) could not reach the cytoplasm for a while. As an extra advantage, $\mathrm{Ca}^{2+}$ in the apoplast would enhance MAMP recognition. It is revealing that none of these processes would be up-regulated in the susceptible genotypes.

\subsubsection{H. brasiliensis striking defense reactions can be self-harmful}

Native proteins in the dark cyan sub-cluster (Table 1, S-C = DCY) at the center of Fig. 1 and its neighbor to the right, the cyan sub-cluster (Table $1, \mathrm{~S}-\mathrm{C}=\mathrm{CY}$ ), were all coded in transcripts up-regulated in $H$. brasiliensis. The same is true for the pink sub-cluster (Table 1, S-C = PK), in the bottom left corner of Fig. 1 and the sandy brown (Table 1, S$\mathrm{C}=\mathrm{SBR}$ ) sub-cluster just beneath. These four sub-clusters consist almost exclusively of proteins that are responsive to JA and all of them 
followed the expression pattern number 1 .

Many native members of the cyan sub-cluster are TIFY/JAZ transcription factors (items $1,6,8,10,13$, and 14 Table 1). TIFY/JAZ protein clusters were not observed among those produced only with proteins up-regulated in the resistant genotypes (Suppl. Mat. S4 $\mathrm{x}$ Suppl. Mat. S5). In addition to RLP1 (item 15), which was identified as a pattern recognition receptor (PRR) that can interact with MAMPs and LCR69 (item 23), which is homologous to PDF1.2, these proteins last mentioned are all activated in response to JA [38,39]. As the exception, the pathogenesis-related protein PR1 (item 11) that participates in more specific downstream reactions is, according to the Arabidopsis locus description, responsive to SA [see 8 too]. Interestingly, PR1 is also involved in the management of excess excitation energy, which causes plastoquinones in the chloroplasts and mitochondria to enter altered redox states, leading to cell death and tissue damage [40]. In addition, the extent of tissue damage can also be related to cyanogenesis intensity [41].

The BOL A protein (item 3, Table 1) is involved in controlling the redox status in chloroplasts or mitochondria as stated in the $A$. thaliana locus AT5G17560 description; the PAT1 protein (item 17, Table 1) is involved in light signal transduction mediated by Phytochrome A and the sieve element occlusion protein, SEOa (item 12, SBR Table 1), is important for the organization of phloem cells to form sieve elements. A. thaliana paralogs SEOR1 and SEOR2 can heterodimerize to each other and drive phloem constriction. In addition, SEOR2 can physically interact with RPM1-RIN4 systems and impair phytoplasma dispersion, regardless of sieve element occlusion. It has been speculated that, by reducing the availability of one of the SEOR paralogs, the other one becomes free from dimerization and available to activate defense responses instead of phloem constriction [42].

To be short, in the susceptible genotypes a second, JA-triggered, defense line against $P$. ulei, would be initiated, following RLP1 upregulation, maybe because the SA-triggered defense mechanisms became mild and arrested, and insufficient to diminish the invasive capacity of the pathogen, despite possibly constitutive in the lack of sufficient CNGC4 (see the Discussion).

\subsubsection{Cell wall reinforcement versus stress attenuation and renovation of the crown}

The light green sub-cluster (Table 1, S-C = LGG) in the upper right corner in Fig. 1 included the native proteins LDOX (item 2) and TT4 (item 16 ), which are up-regulated in $H$. brasiliensis, following expression patter number 1 . Both proteins were found to be consistently associated with a same list of GO terms, such as cellular response to oxidative stress (GO:0006979), response to JA (GO:0009753), and response to wounding (GO:0009611). LDOX is a leucoanthocyanidin synthase that likely participates in vacuole organization as well. The synthesis of anthocyanins in the leaves aids in the protection of photosystems against intense light. TT4 is a chalcone synthase, and chalcone is a precursor for various classes of flavonoids, which are themselves important for the lignification of the cell walls and the synthesis of anthocyanins. Most of the proteins in the light-green sub-cluster are involved in phenylalanine, flavonoid, anthocyanidin, and phenylpropanoid biosynthesis and participate in the reinforcement of the cell wall by lignification, which is a common defense reaction against fungi, one of a few pathogens that can destroy lignin [43].

In the same light-green sub-cluster, the protein encoded in transcript 26, SRG1 (item 36), and PER64 (item 41, Table 1) were found to be up-regulated in $H$. guianensis $+H$. pauciflora, following expression patter number 2. PER64 is a peroxidase specifically involved in the guidance of lignin deposition, a process that is well characterized in the endodermis of roots [44]. SRG1 and MSL10 (see above) genes were both up-regulated in $H$. guianensis $+H$. pauciflora and also were the only two proteins in the whole experiment found to be associated with the GO term "phyllome development" (GO:0090693), meaning "the process whose specific outcome is the progression of a phyllome over time, from its formation to the mature structure. A phyllome is a collective term for all of the different types of leaves appearing on plants", according to the Gene Ontology database. Up-regulation in the evergreens $H$. guianensis and $H$. pauciflora is certainly related to the continuous renovation of the crown, which could employ programmed cell death and controlled senescence, in contrast to $H$. brasiliensis plants that are caducous.

SRG1 is involved in senescence and was very recently associated with a nitric oxide-driven negative feedback that attenuates the plant's immune response. Nitric oxide follows ROS bursts in response to pathogen attack and activates SRG1 transcription. SRG1 would hijack immune response co-repressors, such as TOPLESS, and transiently contribute to enhance defense reactions while promoting its own transcription. However, continuous accumulation of nitric oxide would eventually lead to the nitrosylation of SRG1 itself, which becomes unable to function as a transcription factor. Decreased SRG1 expression or activity results in the accumulation of PR-1 transcripts [45], and this interaction connects the light-green sub-cluster to the cyan and dark cyan sub-clusters (see above). Indeed, continuous ROS and nitric oxyde accumulation could be the cause for the three-fold down-regulation of SRG1 transcription in $H$. brasiliensis relative to $H$. guianensis $+H$. pauciflora (Table 1).

Phenylalanine-, flavonoid-, anthocyanidin-, and phenylpropanoidrelated proteins were not represented in the String clusters for upregulated $H$. guianensis $+H$. pauciflora items only (Suppl. Mat. S5) as strongly as in the clusters for up-regulated transcripts in $H$. brasiliensis (Suppl. Mat. S4). On the contrary, susceptible genotypes did not overexpress proteins dedicated to pectin modification (PMR6 in the blue sub-cluster), or cell wall extension and enhanced flexibility (XTH8 in the brown sub-cluster) or the polygalacturonase inhibitor (PGPI in the brown sub-cluster). This result is interesting because susceptible and resistant genotypes activate defense mechanisms related to the cell wall, but in the resistant genotypes cell wall properties, such as elasticity, stiffness and flexibility [32,33], were assessed while in the susceptible ones, wall thickening driven by a reduction in PMR6 expression [32] and lignification were triggered.

\subsubsection{Cyanogenesis}

The small purple and the yellow sub-clusters (Fig. 1) contain proteins that are committed to the synthesis of glucosinolates and cyanogenic glycosides (GO processes 0019757-0019761 and 0016137-0016139). Glucosinolates evolved from cyanogenic glycosides, and enzymes involved in glucosinolate synthesis would occur in plant species, such as A. thaliana, displaying a sort of ancestral "predisposition" [46]. This supports the use of $A$. thaliana as model for the rubber tree reactions activated in cyanogenesis.

Cyanogenic glycosides are products of the conjugation of aglycon moieties or cyano-amino acids (produced by the conjugation of $\mathrm{HCN}^{-}$ with amino acid residues) with glucose [4]. The most abundant cyanogenic glycoside in rubber trees is linamarin, which is produced in the leaf cell cytoplasm and stored in special vacuoles. Under attack, the contents of the cell vacuoles come in contact with the cytoplasm [47], and specific B-glucosidases remove the glucose moiety producing free aglycon. Then, hydroxynitrile lyases at the plasma membrane break the aglycon to produce free $\mathrm{HCN}^{-}$and aldehyde or ketone [48]. This mechanism keeps cyanide away from the electron transport chains in chloroplasts and mitochondria and makes the leaf exudation produced by the lesions extremely toxic for most pathogens and herbivores, but not for P. ulei [49], as detailed in the Discussion.

The native member of the purple sub-cluster that is up-regulated in H. brasiliensis was inscribed as the valine monooxygenase 1 in $\mathrm{H}$. brasiliensis reference genome (Table 1 , item $4, \mathrm{~S}-\mathrm{C}=\mathrm{PRP}$ ). The cyanoamino acids derived from valine through the reaction catalyzed by the valine monooxygenase are precursors of aglycons used to produce linamarin (and lotaustralin) upon glycosylation (Suppl. Mat. S7). Indeed, locus 110641550 codes for a peptide that presents better homology to 
L110639362 RUBBER TREE gi249261 Manihot esculenta gi1126497856 populus tomentosa gi1126497860 populus tomentosa

L110639362 RUBBER TREE gi249261 Manihot esculenta gi1126497856 populus tomentosa gi1126497860 Populus tomentosa

L110639362 RUBBER TREE gi249261 Manihot esculenta gi1126497856 populus tomentosa gi1126497860 Populus tomentosa

L110639362 RUBBER TREE gi249261 Manihot esculenta gi1126497856 populus tomentosa gi1126497860 populus tomentosa

L110639362 RUBBER TREE gi249261 Manihot esculenta gi1126497856 Populus tomentosa gi1126497860 populus tomentosa

L110639362 RUBBER TREE gi249261 Manihot esculenta gi1126497856 Populus tomentosa gi1126497860 populus tomentosa

L110639362 RUBBER TREE gi249261 Manihot esculenta gi1126497856 populus tomentosa gi1126497860 populus tomentosa

L110639362 RUBBER TREE gi249261 Manihot esculenta gi1126497856 populus tomentosa gi1126497860 populus tomentosa
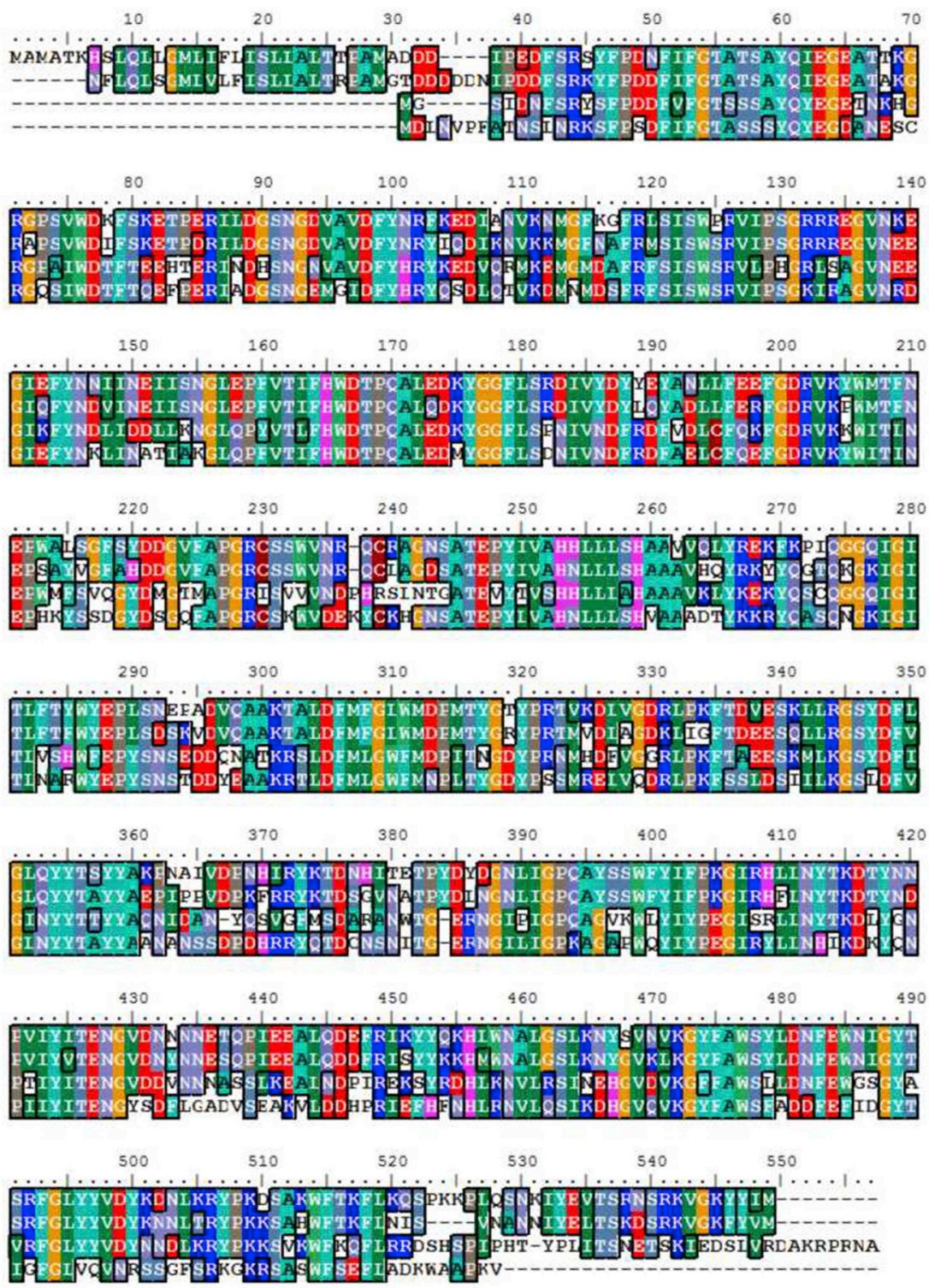

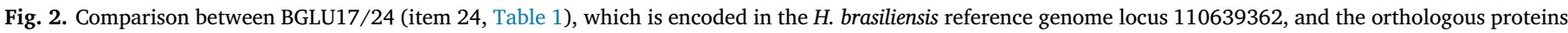

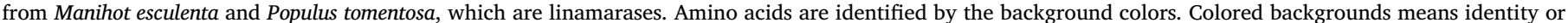
similarity. The GI in the sequence titles are the accession numbers for the genes in GenBank.

valine monooxygenases from Manihot esculenta, including CYP79D2 [50], and from Ricinus communis which are cyanogenic species, than to the CYP79B2 from Arabidopsis, which is a monooxygenase that uses tryptophan as substrate for the cyano-amino acid production (Suppl. Mat. S7). Nevertheless, the sequence for the Arabidopsis CYP79D2 is not available in the Arabidopsis databases. In this reason, the CYP79B2, the best homolog available for the rubber tree peptide encoded in transcript 4 was hit by the BioSystems.

The native member of the purple sub-cluster that was up-regulated in the resistant genotypes $H$. guianensis $+H$. pauciflora is an ortholog of the A. thaliana CYP81D1 (Table 1 , item 42, SC = PRP). The only CYP81D class member in $H$. brasiliensis is a CYP81D11, and most of the CYP81 family members are currently included in the CYP81E subclass. Based on its homology to the Arabidopsis enzyme and the association with the light-green sub-cluster, CYP81D1 is a monooxygenase (cytochrome P450) that could be primarily involved in isoflavonoid biosynthesis, which is also inducible and may be defense related [51], (Suppl.Mat.S8). Nevertheless it was automatically included in the purple sub-cluster, which is populated with proteins involved in cyanogenesis. Cyanogenesis and phenylpropanoid biosynthesis and accumulation can interfere with each other [52], and this CYP81D1 monooxygenase could play a role in the modulation of defense by cyanogenesis versus growth by the production of cell wall precursors. Alternatively, the rubber tree CYP81D1 ortholog may be enrolled in cyanogenesis only, but it has not yet been sufficiently studied or characterized in rubber trees or other cyanogenic plant species.

In the yellow sub-cluster, BGLU17/24 (encoded in transcript 24, $\mathrm{S}-\mathrm{C}=\mathrm{Y}$ in Table 1 ) is up-regulated in $H$. brasiliensis. The peptide deduced from transcript 24 is extremely similar to linamarases from Manihot esculenta and Populus tomentosa (Fig. 2), which also produce linamarin [43]. The second protein in the yellow sub-cluster is MES5 (encoded in transcript number 44, S-C $=\mathrm{Y}$ in Table 1 ), which is upregulated in the resistant genotypes $H$. guianensis $+H$. pauciflora. According to its homology to Manihot esculenta and Populus tomentosa proteins, MES5 is a hydroxynitrile lyase (see also Suppl. Mat. S9), acting in the last step of cyanogenesis, when the aglycon is broken and $\mathrm{HCN}^{-}$ is released from injured cells [4].

Indeed, taking into account CYP79D2 and BGLU17/24 up- 
regulation, the increase in linamarin production and the release of aglycons from linamarin could be, respectively, 5 and 1.5 folds higher in $H$. brasiliensis (Table 1 , items 4 and 24). However, these two processes would not be coordinated with the increase in $\mathrm{HCN}^{-}$release from the aglycons that results from MSE5 lyase action. MSE5 lyase (Table 1, item 44) expression is almost 5 folds lower in $H$. brasiliensis when contrasted to $H$. guianensis $+H$. pauciflora, while CYP79D2 and BGLU17/24 (Table 1, items 4 and 24) are down-regulated in these two resistant species. Interestingly, these results did not disagree with those already reported about the cyanogenic capacity and the cyanogenic potential in resistant and susceptible rubber trees (see Discussion).

\subsubsection{Histones and nucleosome organizers}

Finally, the green sub-cluster of proteins, down in Fig. 1, is vastly dominated by histones and nucleosome organizers. Only one native protein encoded by a transcript included in Table 1 joined this subcluster: the H2B-like histone, which is ortholog to HBT4 from $A$. thaliana (item $45, \mathrm{G}$ in Table 1 ) and is up-regulated in $H$. guianensis $+H$. pauciflora. The vast majority of proteins in this green subcluster were, indeed, tracked from the String databases by item 45 , and are connected to proteins in the lime green, dark cyan, and red subclusters due to the interactions between ubiquitination, the cell cycle, and processes occurring in the chloroplasts, including chloroplast regeneration and ribosome assembly. The modification of histones can be part of defense strategies based in chromatin remodeling [7] and would be active just in resistant genotypes.

\subsection{Polymorphisms identified in differentially expressed genes}

Comparing the structures of paralogs from the different Hevea species for the 45 loci in Table 1, approximately 2,031 polymorphic sites were identified, 1,723 of which were SNPs and 308 of which were microsatellites differing in the number of repeats in their core or in insertions/deletions of a few bases not associated with the microsatellite cores (Suppl. Mat. S10).

Significantly, most of the polymorphisms diverged consistently when genotypes susceptible to $P$. ulei were compared to the tolerant/ resistant genotypes. These findings supported the strategies applied to select the transcripts in Table 1. Some of the polymorphic sites will be described in the following paragraphs.

Transcripts encoded in the paralogs to the $H$. brasiliensis locus 110639465 , which code for the CIPK14, are plenty of polymorphic sites (Fig. 3). CIPK14 (item 27 in Table 1, S-C = B) is involved in $\mathrm{Ca}^{2+}$ flow. The presence of higher amounts of reads coming from the resistant genotypes $H$. guianensis and $H$. pauciflora in comparison to reads from $H$. brasiliensis susceptible genotypes (clones RRIM 600 and RRIM 713), which can be estimated by the height of the lines in the different colors, was remarkable (Fig. 3a) and is in agreement with the fold-change values included in Table 1.

In addition, the alleles observed in the resistant genotypes are the same as those observed in the moderately resistant $H$. brasiliensis clone CNS AM 7905 (Fig. 3b and c). Different alleles were found to be consistently related to the different plant phenotypes, and divergences were observed in the regions coding for ATP binding sites (Fig. 3b) and for the site of interaction with CBL proteins (Fig. 3c, Suppl. Mat. S11).

Additional interesting examples of polymorphic sites strongly related to the rubber tree phenotypes of SALB resistance or susceptibility are available as Suppl. Mat. S12, for loci 110657526 and 110639362 , coding for the AOR (item 30, Table 1) and the BGLU17/24 linamarase (item 24, Table 1), respectively.

\section{Discussion}

The strategy used to analyze the RNA-seq libraries was to contrast the databases from one set of susceptible genotypes $(H$. brasiliensis clones) with a set of resistant genotypes (H. guianensis $+H$. pauciflora clones), in order to select genes displaying differential expression patterns consistently related to resistance or susceptibility to SALB. Indeed, concerning those genes, the expression pattern in five $H$. guianensis $+H$. pauciflora SALB resistant clones taken together was expected to be opposite to the pattern observed for the two $H$. brasiliensis clones that were susceptible to SALB. The combined action of the genes up and down-regulated to produce the contrasting phenotypes is discussed below and supports the hypothesis that sampled plants were all challenged by $P$. ulei, because SALB resistance or susceptibility was the only criterion used to organize the different rubber tree RNA-seq databases, as a preparation for the differential expression analysis, and the differences observed followed the expections.

In $H$. brasiliensis susceptible genotypes, the down-regulation of LRK1 and ATL2/RIN2 and MSL10 would delay MAMP recognition in young leaves, facilitating the occurrence of epidermal cell necrosis [2] and the evolution of the initial abaxial conidial lesions in stages $\mathrm{B} / \mathrm{C}$ to the adaxial mature pycnidia in stages $\mathrm{C} / \mathrm{D}$ [53]. A reduced expression of the polygalacturonase inhibitor (PGPI), which is allocated in the cell walls to decelerate pathogen invasion [34], contributed to ease the colonization process and to trigger JA responsive pathways. In these susceptible genotypes the pathogen is supposed to have reached the epidermal cell cytoplasm and, maybe, even the vascular bundles, judging by the up-regulation of PR1 [7] and SEOa [42]. And it is intriguing that investing in cell wall lignification and thickening was not effective (but see below). At this point, the activation of JA-triggered pathways was, probably, initiated by RLP1, which is encoded in the only MAMP-recognition gene up-regulated in susceptible genotypes, and mediated by LCR69, which is an ortholog to PDF1.2 $[38,39]$. The up-regulation of these two last genes in addition to four TIFY/JAZ proteins which are all responsive to JA and the simultaneous up-regulation of the $P R 1$ ortholog that responds to SA $[7,8]$, indicated that SA and JA-responsive pathways were active in susceptible genotypes. SA-JA cross-talk and synergy has already been documented $[8,54]$. And so, despite delayed as a consequence of deficient LRK1, ATL2/RIN2 and MSL10 transcription, which was 3.9, 1.8 and 2.9 folds lower, respectively, in susceptible genotypes, SA responsive reactions would be active, enhancing cell death, due to the 1.8 folds lower expression of the CNGC4 gene $[19,20]$. This "state of war" combined with cyanogenesis and direct exposure to the high light intensities of equatorial latitudes could disturb and conduce organelles to disassemble $[38,40,41,55]$ and proteins to enter ubiquitination and recycling $[25,26]$, thus accelerating the natural predisposition of $H$. brasiliensis toward seasonal leaf senescence.

Resistant genotypes $H$. guianensis $+H$. pauciflora, however, could display precisely controlled and rapidly triggered SA-responses following pathogen recognition, accomplished by LRK1 and/or by ATL2/ RIN2 partners, which had the expression 3.9 and 1.8 folds increased, respectively, in resistant genotypes. The induction of $\mathrm{HR}$ under the control of CHGC4 [19,20], which was also up-regulated, indicated the activation of SA-triggered responses. The deposition of delicate lignin strips associated with PER64 activity in internal leaf cellular rows [44,56], and the up-regulation of PGIP1-driven fungal-polygalacturonase inhibition, both would impair the SALB spreading, even when the PMR6 was up-regulated too. The PMR6 pectate lyase can function as a "susceptibility factor", but up-regulation in the resistant genotypes would, simultaneously, contribute to maintain the cell wall flexible and able to extend, grant the contact of the fungi with the PGIP as soon as possible, and assure a long term up-regulation of MSL10, which encodes a stress-related mechano-sensitive channel able to recognize wounding [22,23], in young leaves that are expanding. Meanwhile, oxidative and osmotic stresses, in the cytoplasm and the organelles, would decrease under up-regulated $\mathrm{Ca}^{2+}$ trafficking and nitric oxide related mechanisms controlled by SRG1 [45,57], providing the conditions to withstand $P$. ulei and escape leaf senescence and decay. $\mathrm{Ca}^{2+}$ and nitric oxide could both induce JA related processes [7]. Nevertheless, JA responsiveness would be under SA control in resistant genotypes. NPR1 is necessary to stop JA-triggered responses and 


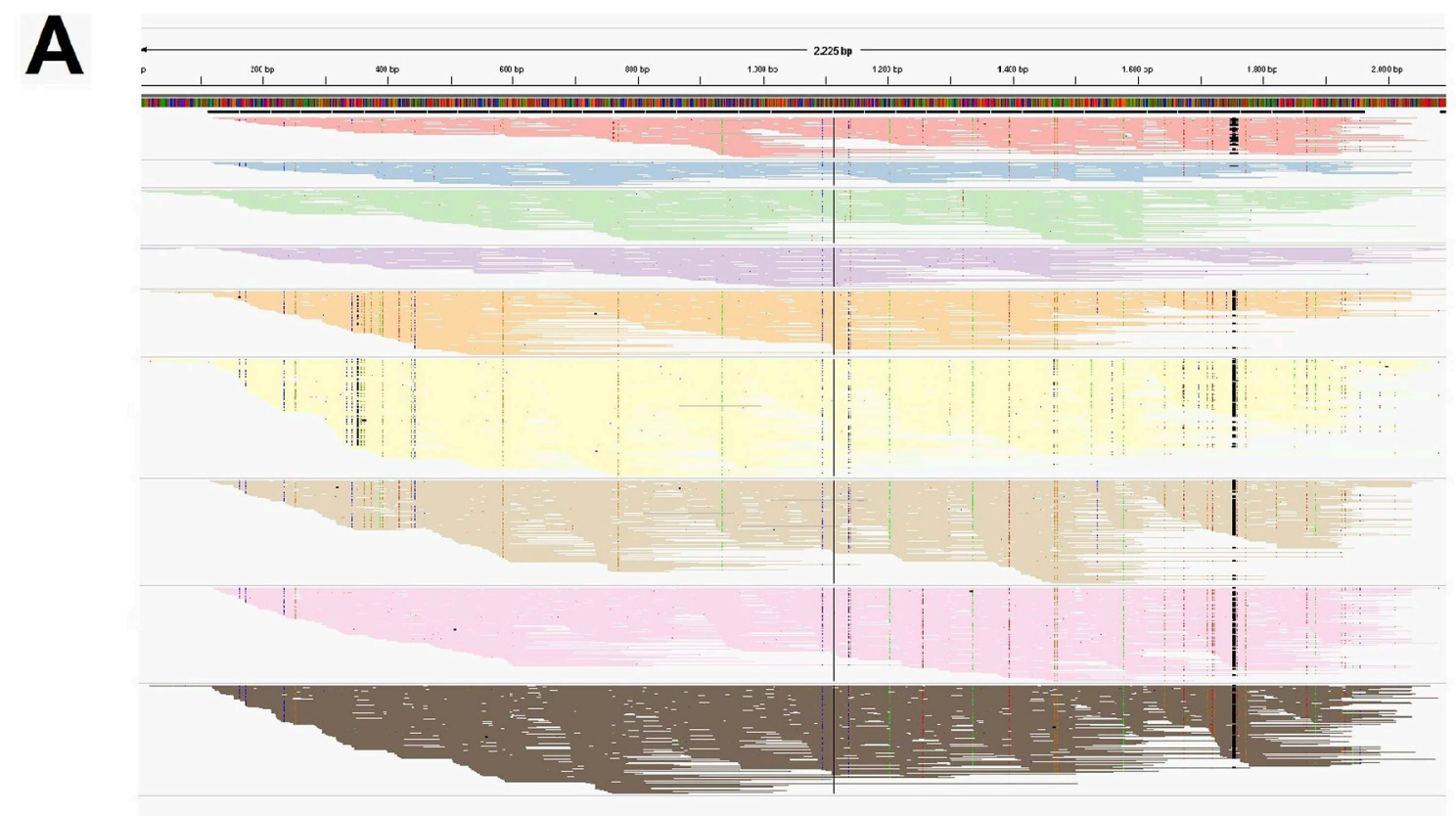

B
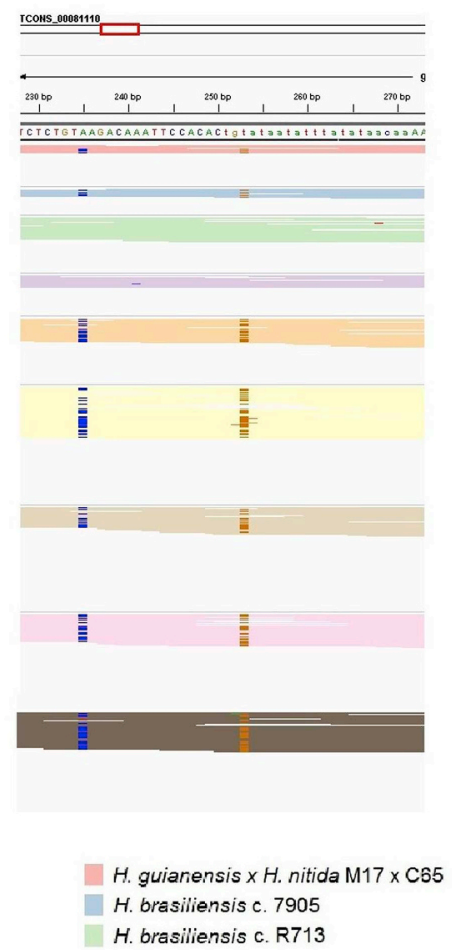
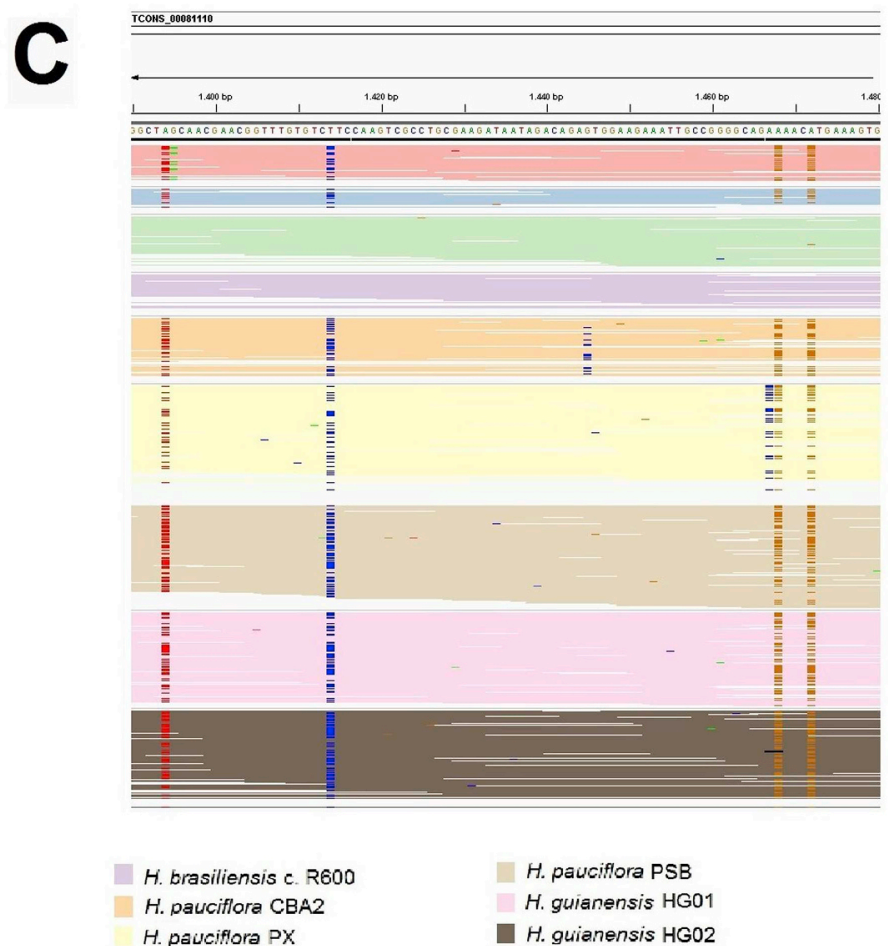

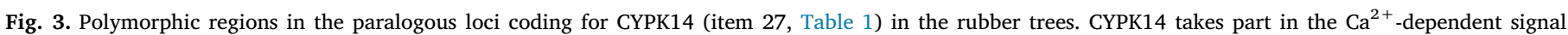

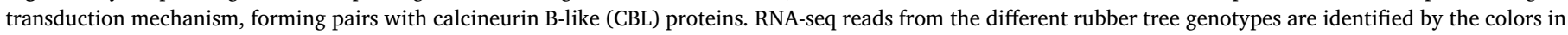

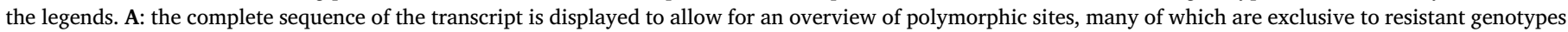

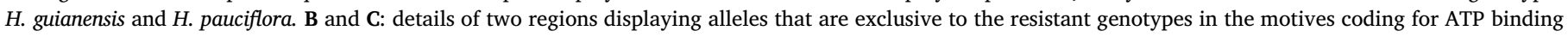

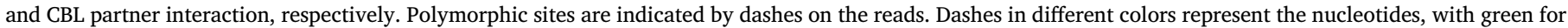

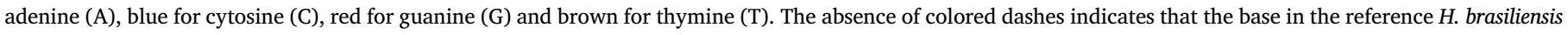
locus 110639465 was preserved, as indicated at the top of the figure by the letters representing the nucleotides.

it is responsive to SA [7]. Since it was not present among the DEGs maybe NPR1 was equally expressed in resistant and susceptible genotypes at the moment of the collects. Indeed the superimposed operation of JA and SA-triggered responses in susceptible genotypes could be imputed, as mentioned above, to the delay in the initial MAMP-recognition, leading to the delay in NPR1 up-regulation in susceptible plants, while epidermal cell necrosis and tissue invasion were taking place $[2,49,53]$.

Regarding cyanogenesis specifically, the resistant genotypes expressed the monoxygenase CYP81D1 and the MES5 lyase orthologs in higher amounts, while the susceptible genotypes over-expressed the monoxygenase CYP79B2 and the linamarase BGLU17/24 orthologs. Indeed, as already reported, susceptible genotypes can display higher cyanogenic potential (the preparation and storage of cyanogenic 
precursors) and, simultaneously, lower cyanogenic capacity due to the lower or slower $\mathrm{HCN}^{-}$release in the extracellular spaces [4], which is, judging by the results presented herein, at least in part, a consequence of insufficient MES5 hydroxynitrile lyase gene transcription. Anyway, considering the accumulation of high contents of linamarin in cells/ tissues, which can be inflated in response to JA [58], even mild MES5 lyase activity could lead to, slow but constant and cumulative production of free $\mathrm{HCN}^{-}$in susceptible genotypes.

In contrast, the SALB resistant $H$. guianensis and $H$. pauciflora would have a lower cyanogenic potential [4] due to decreased CYP79B2 monooxygenase and BGLU17/24 B-glucosidase expression, but extracellular $\mathrm{HCN}^{-}$would be rapidly released, as soon as the over-expressed MSE5 lyase could access some aglycon. In addition, $\mathrm{HCN}^{-}$can reportedly be produced by the natural decay of the aglycon, particularly at alkaline $\mathrm{pH}$ [4], which could, possibly, be granted by the trafficking of $\mathrm{Ca}^{2+}$ to the apoplast, and young P. ulei mycelia have been found to present better vegetative growth in the presence of $\mathrm{HCN}^{-}$than in its absence [49]. Indeed, resistance would relay on the equilibrium among vigilance, internal stress control, maintenance of housekeeping processes that support growth and the provision of a comfortable environment for the pathogen outside the cells, which would also contribute to prevent sporulation [49] and the colonization of cellular inner spaces.

The differences between the two physiological conditions - resistance or susceptibility - were also supported by the identification of contrasting allele configurations that prevailed in one or the other. The $H$. brasiliensis clone CNS AM 7905, which has been classified as moderately resistant to $P$. ulei in field observations, as well as the $H$. guianensis $\mathrm{x} H$. nitida hybrid, were distinguished by their "blended" or hybrid haplotypes. The gene sequence configurations can interfere in transcript accumulation and/or protein effectiveness, thus contributing directly to define resistant or susceptible phenotypes. The analysis of segregation and validation of the polymorphisms identified herein will make it possible for some to be used as genetic markers and to aid in the selection of resistant plants. Finally, the other 282 DEGs, some of them possibly related to defense reactions as well, must be examined (Suppl. Mat. S13).

\section{CRediT authorship contribution statement}

Paula Cristina da Silva Angelo: Conceptualization, Investigation, Formal analysis, Data curation, Writing - original draft, Writing - review \& editing. Michel Eduardo Beleza Yamagishi: Investigation, Formal analysis, Data curation, Writing - original draft, Writing - review \& editing. Jeferson Chagas da Cruz: Investigation. Gilvan Ferreira da Silva: Project administration, Investigation, Writing - review \& editing. Luadir Gasparotto: Investigation, Writing - review \& editing.

\section{Declaration of competing interest}

Authors inform that there is no conflict of interest regarding the publication of these data.

\section{Acknowledgments}

This research was supported by the Brazilian Agricultural Research Corporation - Embrapa (grant \# 02.13.07.002.08.009). The authors are thankful to Dr. Brad Day, while Editor in Chief, and to the anonymous reviewers which gave us the opportunity to produce a better text, to Dr. José Clério Rezende Pereira (in memoriam) for the instigating dialogues, and to Dr. Larissa Alexandra Cardoso Moraes and Dr. Rivadalve Coelho Gonçalves for their critical reading of the manuscript. Dr. Michel E. B. Yamagishi acknowledges the LMB (Laboratório Multiusuário de Bioinformática da EMBRAPA) for the computational structure provided.

\section{Appendix A. Supplementary data}

Supplementary data to this article can be found online at https:// doi.org/10.1016/j.pmpp.2020.101477.

\section{References}

[1] V.H.F. Moraes, L.A.C. Moraes, Desempenho de clones de copa de seringueira resistentes ao mal-das-folhas, Pesqui. Agropecuária Bras. 43 (2008) 1495-1500.

[2] L. Gasparotto, F.A. Ferreira, A.F. dos Santos, J.C.R. Pereira, E.L. Furtado, Mal-dasfolhas, in: Gasparotto, Pereira (Eds.), Doenças da seringueira no Brasil, 2012, pp. 37-94 Embrapa Amazônia Ocidental.

[3] R.J. Seibert, A study of Hevea (with economical aspects) in the Republic of Peru, Ann. Mo. Bot. Gard. 3 (1947) 259-352.

[4] R. Lieberei, Relationship of cyanogenic capacity (HCN-c) of the rubber tree Hevea brasiliensis to susceptibility to Microcyclus ulei, the agent causing South American Leaf Blight, J. Phytopathol. 122 (1988) 54-67.

[5] A.Y.A. Rahman, A.O. Usharraj, B.B. Misra, G.P. Thottathil, K. Jayasekaran, Y. Feng, S. Hou, S.Y. Ong, F.L. Ng, L.S. Lee, H.S. Tan, M.K.L.M. Sakaff, B.S. Teh, B.F. Khoo, S.S. Badai, N.A. Aziz, A. Yuryev, B. Knudsen, A. Dionne-laporte, N.P. Mchunu, Q. Yu, B.J. Langston, T.A.K. Freitas, A.G. Young, R. Chen, L. Wang, N. Najimudin, J.A. Saito, M. Alam, Draft genome sequence of the rubber tree Hevea brasiliensis, BMC Genom. 14 (2013) 75.

[6] U.A. Hurtado Páez, I.A. García Romero, S. Restrepo Restrepo, F.A. Aristizábal Gutiérrez, D. Montoya Castaño, Assembly and analysis of differential transcriptome responses of Hevea brasiliensis on interaction with Microcyclus ulei, PloS One 10 (2015) e01348377.

[7] J.S. Ramirez-Prado, A.A. Abulfaraj, N. Rayapuram, M. Benhamed, H. Hirt, Plant immunity: from signaling to epigenetic control of defense, Trends Plant Sci. 23 (2018) 833-844.

[8] Z. Chen, J. Wang, Y. Li, Y. Zhong, J. Li, S. Lu, L. Wang, X. Wang, S. Chen, Dry mycelium of Penicillium chrysogenum activates defense via gene regulation of salicylic acid and jasmonic acid signaling in Arabidopsis, Physiol. Mol. Plant Pathol. 103 (2018) 54-61.

[9] D. Kim, G. Pertea, C. Trapnell, H. Pimentel, R. Kelley, S.L. Salzberg, TopHat2: accurate alignment of transcriptomes in the presence of insertions, deletions and gene fusions, Genome Biol. 14 (2013) R36.

[10] C. Trapnell, A. Roberts, L. Goff, G. Pertea, D. Kim, D.R. Kelley, H. Pimentel, S.L. Salzber, J.L. Rinn, L. Pachter, Differential gene and transcript expression analysis of RNA-seq experiments with TopHat and Cufflinks, Nat. Protoc. 7 (2012) 562-578.

[11] C. Trapnell, D.G. Handrichson, M. Sauvageau, L. Goff, J.L. Rinn, L. Pachter, Differential analysis of gene regulation at transcript resolution with RNA-seq, Nat. Biotechnol. 31 (2013) 46.

[12] BioSystems, www.ncbi.nlm.nih.gov/biosystems.

[13] TAIR, www.arabidopsis.org.

[14] Gene Ontology (GO), www.geneontology.org.

[15] String, www.string-db.org.

[16] S. Dongen, Graph clustering via a discrete uncoupling process, SIAM J. Matrix Anal. Appl. 30 (2008) 121-141.

[17] A. Köhler, E. Hurt, Exporting RNA from the nucleus to the cytoplasm, Nat. Rev. Mol. Cell Biol. 8 (2007) 761-773.

[18] I. Ohbayashi, M. Sugiyama, Plant nucleolar stress response, a new face in the NACdependent cellular stress responses, Front. Plant Sci. 8 (2018) 2247.

[19] C. Balagué, B. Lin, C. Alcon, G. Flottes, S. Malmström, C. Köhler, G. Neuhaus, G. Pelletier, F. Gaymard, D. Roby, HLM1, an essential signaling component in the hypersensitive response, is a member of the cyclic nucleotide-gated channel ion channel family, Plant Cell 15 (2003) 365-379.

[20] K. Chin, T.A. DeFalco, W. Moeder, K. Yoshioka, The Arabidopsis cyclic nucleotidegated ion channels AtCNGC2 and AtCNGC4 work in the same signaling pathway to regulate pathogen defense and floral transition, Plant Physiology 163 (2013) 611-624.

[21] Y. Yamauchi, A. Hasegawa, M. Mizutani, Y. Sugimoto, Chloroplastic NADPH-dependent alkenal/one oxidoreductase contributes to the detoxification of reactive carbonyls produced under oxidative stress, FEBS (Fed. Eur. Biochem. Soc.) Lett. 586 (2012) 1208-1213.

[22] Y. Zou, S. Chintamanani, P. He, H. Fukushige, L. Yu, M. Shao, L. Zhu, D.F. Hildebrand, X. Tang, J.-M. Zhou, A gain-of-function mutation in MSL10 triggers cell death and wound-induced hyper-accumulation of jasmonic acid in Arabidopsis, J. Integr. Plant Biol. 58 (2016) 600-609.

[23] K.M. Veley, G. Maksaev, E.M. Frick, E. January, S.C. Kloepper, E.S. Haswel, Arabidopsis MSL10 has a regulated cell death signaling activity that is separable from its mechanosensitive ion channel activity, Plant Cell 26 (2014) 3115-3131.

[24] K. Bouwmeester, F. Govers, Arabidopsis L-type lectin receptor kinases: phylogeny, classification, and expression profiles, J. Exp. Bot. 60 (2009) 4383-4396.

[25] B. Zhou, L. Zeng, Conventional and unconventional ubiquitination in plant immunity, Mol. Plant Pathol. 18 (2017) 1313-1330.

[26] F. Li, R.D. Vierstra, Autophagy: a multifaceted intracellular system for bulk and selective recycling, Trends Plant Sci. 17 (2012) 1360-1385.

[27] M. Libault, J. Wan, T. Czechowski, M. Udvardi, G. Stacey, Identification of 118 Arabidopsis transcription factors and 30 ubiquitin-ligase genes responding to chitin, a plant-defense elicitor, Mol. Plant Microbe Interact. 20 (2007) 900-911.

[28] P. Kapos, K.T. Devendrakumar, X. Lia, Plant NLRs: from discovery to application, Plant Sci. 279 (2019) 3-18. 
[29] T. Kawasaki, J. Nam, D.C. Boyes, B.F. Holt, D.A. Hubert, A. Wiig, J.L. Dang, A duplicated pair of Arabidopsis RING-finger E3 ligases contribute to the RPM1- and RPS2-mediated hypersensitive response, Plant J. 44 (2005) 258-270.

[30] Y. Kanno, Y. Kamiya, M. Seo, Nitrate does not compete with abscisic acid as a substrate of AtNPF4.6/NRT1.2/AIT1 in Arabidopsis, Plant Signal. Behav. 8 (2013) e26624.

[31] D. Ré, M. Capella, G. Bonaventure, R.L. Chan, Arabidopsis AtHB7 and AtHB12 evolved divergently to fine tune processes associated with growth and responses to water stress, BMC Plant Biol. 14 (2014) 150.

[32] T. Engelsdorf, C. Will, J. Hofmann, C. Schmitt, B.B. Merritt, L. Rieger, M.S. Frenger, A. Marschall, R.B. Franke, S. Pattathil, L.M. Voll, Cell wall composition and penetration resistance against the fungal pathogen Colletotrichum higginsianum are affected by impaired starch turnover in Arabidopsis mutants, J. Exp. Bot. 68 (2017) 701-713.

[33] R. Balsamo, M. Boak, K. Nagle, B. Peethambaran, B. Layton, Leaf biomechanical properties in Arabidopsis thaliana polysaccharide mutants affect drought survival, J. Biomech. 48 (2015) 4124-4129.

[34] C.-X. Di, H. Zhang, Z.-L. Sun, H.-L. Jia, L.-N. Yang, J. Si, L.-Z. An, Spatial distribution of polygalacturonase-inhibiting proteins in Arabidopsis and their expression induced by Stemphylium solani infection, Gene 506 (2012) 150-155.

[35] O. Batistic, R. Waadt, L. Steinhorst, K. Katrin Held, K. Jörg, CBL-mediated targeting of CIPKs facilitates the decoding of calcium signals emanating from distinct cellular stores, Plant J. 61 (2010) 211-222.

[36] R.D. Hoffmann, L.I. Olsen, C.V. Ezike, J.T. Pedersen, R. Manstretta, R.L. LópezMarqués, M. Palmgren, Roles of plasma membrane proton ATPases AHA2 and AHA7 in normal growth of roots and root hairs in Arabidopsis thaliana, Physiol. Plantarum 166 (2018) 12842.

[37] E. Lam, N. Kato, M. Lawton, Programmed cell death, mitochondria and the plant hypersensitive response, Nature 411 (2001) 848-852.

[38] J. Monaghan, F. Xu, M. Gao, Q. Zhao, K. Palma, C. Long, S. Chen, Y. Zhang, X. Li, Two Prp19-Like U-Box proteins in the MOS4-Associated Complex play redundant roles in plant innate immunity, PLoS Pathog. 5 (2009) e1000526.

[39] A.K. Jehle, U. Fürst, M. Lipschis, M. Albert, G. Felix, Perception of the novel MAMP eMax from different Xanthomonas species requires the Arabidopsis receptor-like protein ReMAX and the receptor kinase SOBIR, Plant Signal. Behav. 8 (2013) e27408.

[40] P. Mühlenbock, M. Szechynska-Hebda, M. Płaszczyca, M. Baudo, P.M. Mullineaux, J.E. Parker, B. Karpinska, S. Karpinskie, Chloroplast signaling and LESION SIMULATING DISEASE1 regulate crosstalk between light acclimation and immunity in Arabidopsis, Plant Cell 20 (2008) 2339-2356.

[41] D. Kadow, K. Voß, D. Selmar, R. Lieberei, The cyanogenic syndrome in rubber tree Hevea brasiliensis: tissue-damage-dependent activation of linamarase and hydroxynitrile lyase accelerates hydrogen cyanide release, Ann. Bot. 109 (2012) 1253-1262.

[42] L. Pagliari, S. Buoso, S. Santi, A.J.E.V. Bel, R. Musetti, What slows down phytoplasma proliferation? Speculations on the involvement of AtSEOR2 protein in plant defence signalling, Plant Signal. Behav. 13 (2018) e1473666.

[43] F.K. Gleason, F.K. Gleason, R. Chollet (Eds.), Plant Biochemistry, Jones and Bartlett Learning, Sudbury - MA. USA, 2012, p. 240.

[44] R.B. Franke, Caspary's conductor, Proc. Natl. Acad. Sci. Unit. States Am. 112 (2015) 10084-11008.

[45] B. Cui, Q. Pan, D. Clarke, M.O. Villarreal, S. Umbreen, B. Yuan, W. Shan, J. Jiang, G.J. Loake, S-nitrosylation of the zinc finger protein SRG1 regulates plant immunity, Nat. Commun. 9 (2018) 4226.

[46] M.D. Mikkelsen, C.H. Hansen, U. Wittstock, B.A. Halkier, Cytochrome P450 CYP79B2 from Arabidopsis catalyzes the conversion of tryptophan to indole-3acetaldoxime, a precursor of indole glucosinolates and indole-3-acetic acid, J. Biol. Chem. 275 (2000) 33712-33717.

[47] E.D. Faÿ, L.A.C. Moraes, V.H.D.F. Moraes, Cyanogenesis and the onset of tapping panel dryness in rubber tree, Pesqui. Agropecuária Bras. 12 (2010) 1372-1380.

[48] D. Selmar, Transport of cyanogenic glucosides: linustatin uptake by Hevea cotyledons, Planta 191 (1993) 191-199.

[49] R. Lieberei, Physiological characteristics of Microcyclus ulei (P. Henn.)v. Arx. - a fungal pathogen of the cyanogenic host Hevea brasiliensis, J. Appl. Bot. Food Qual. 80 (2006) 63-68.

[50] K. Jørgensen, S. Bak, P.T. Busk, C. Sørensen, C.E. Olsen, J. Puonti-Kaerkas, B.L. Møller, Cassava plants with a reduced cyanogenic glucoside content in leaves and tubers. Distribution of cyanogenic glucosides, the site of synthesis and transport, and blockage of the biosynthesis by RNA interference technology, Plant Physiology 139 (2005) 363-374.

[51] T. Akashi, T. Aoki, S.-I. Ayab, CYP81E1, a cytochrome P450 cDNA of Licorice (Glycyrrhiza echinata L.), encodes isoflavone 29-Hydroxylase, Biochem. Biophys. Res. Commun. 251 (1998) 67-70.

[52] J.I. Kim, W.L. Dolan, N.A. Anderson, C. Chapple, Indole glucosinolate biosynthesis limits phenylpropanoid accumulation in Arabidopsis thaliana, Plant Cell 27 (2015) 1529-1546.

[53] B.T. Hora Júnior, D.M. de Macedo, R.W. Barreto, H.C. Evans, C.R.R. Mattos, et al., Erasing the past: a new identity for the damoclean pathogen causing South American Leaf Blight of rubber, PloS One 9 (2014) e104750.

[54] W. Zhang, F. Zhao, L. Jiang, C. Chen, L. Wu, Z. Liu, Different pathogen defense strategies in Arabidopsis: more than pathogen recognition, Cells 7 (2018) 252.

[55] Y. Niwa, T. Kato, S. Tabata, M. Seki, M. Kobayashi, K. Shinozaki, Y. Moriyasu, Disposal of chloroplasts with abnormal function into the vacuole in Arabidopsis thaliana cotyledon cells, Protoplasma 223 (2004) 229-232.

[56] Y. Lee, M.C. Rubio, J. Alassimone, N. Geldner, A mechanism for localized lignin deposition in the endodermis, Cell 153 (2013) 402-412.

[57] Y. Ma, Y. Zhao, R.K. Walker, G.A. Berkowitz, Molecular Steps in the Immune Signaling pathway evoked by plant elicitor peptides: $\mathrm{Ca}^{2+}$-dependent protein kinases, nitric oxide, and reactive oxygen species are downstream from the early $\mathrm{Ca}^{2+}$ signal, Plant Physiology 163 (2013) 1459-1471.

[58] S. Kautz, J.A. Trisel, D.J. Ballhorn, Jasmonic acid enhances plant cyanogenesis and resistance to herbivory in lima bean, J. Chem. Ecol. 40 (2014) 1186-1196. 